\title{
SOME RELATIONS ON KONHAUSER MATRIX POLYNOMIALS
}

\author{
AYMAN SHEHATA
}

Received 10 February, 2014

\begin{abstract}
This paper deals with the study of the generalized hypergeometric matrix function and obtains some of its properties. We rephrase some results from the previous (earlier) works that will be used in this study. We get the hypergeometric matrix function representation, matrix differential equation, generating matrix functions, bilinear generating matrix functions, matrix recurrence relations, finite summation formulas and related results for the Konhauser matrix polynomials given in [34]. Finally, we give some important results involving properties of Mittag-Leffler and Bessel-Maitland matrix functions as applications.
\end{abstract}

2010 Mathematics Subject Classification: 33C45; 33C50; 33C25; 15A60; 33E20; 33C47

Keywords: matrix polynomials, generalized hypergeometric matrix function, integral representation, Konhauser matrix polynomials, generating matrix functions, matrix differential equation, finite summation

\section{INTRODUCTION}

In mathematics, the Konhauser polynomials, introduced by Konhauser [20], are biorthogonal polynomials for the distribution function of the Laguerre polynomials $[23,24]$. Special matrix functions are very closed to statistics, Lie group theory and number theory are well known in $[6,10,22]$. In the recent work, matrix polynomials have significant emergent in $[1-3,5,8,11,12,14-19,21,26-31,33,35]$. Results in the theory of classical orthogonal polynomials have been extended to orthogonal matrix polynomials in $[4,13]$. Hence we feel that the expansions of some functions in terms of the family of Konhauser matrix polynomials $Z_{n}^{A}(x ; k)$ may be of interest to their intrinsic mathematical importance and to the fact that these polynomials have applications in physics.

Throughout this paper, for a matrix $A$ in $\mathbb{C}^{N \times N}$, its spectrum $\sigma(A)$ denotes the set of all eigenvalues of $A$. The two-norm will be denoted by $\|A\|_{2}$ and it is defined by (see [17])

$$
\|A\|_{2}=\sup _{x \neq 0} \frac{\|A x\|_{2}}{\|x\|_{2}},
$$


where for a vector $x$ in $\mathbb{C}^{N},\|x\|_{2}=\left(x^{T} x\right)^{\frac{1}{2}}$ is the Euclidean norm of $x$. Let us denote the real numbers $M(A)$ and $m(A)$ as in the following

$$
M(A)=\max \{\operatorname{Re}(z): z \in \sigma(A)\} ; \quad m(A)=\min \{\operatorname{Re}(z): z \in \sigma(A)\} .
$$

If $f(z)$ and $g(z)$ are holomorphic functions of the complex variable $z$, which are defined in an open set $\Omega$ of the complex plane, and $A, B$ are matrices in $\mathbb{C}^{N \times N}$ with $\sigma(A) \subset \Omega$ and $\sigma(B) \subset \Omega$, such that $A B=B A$, then from the properties of the matrix functional calculus in [9], it follows that

$$
f(A) g(B)=g(B) f(A) .
$$

Throughout this study, a matrix polynomial of degree $n$ in $x$ means an expression of the form

$$
P_{n}(x)=A_{n} x^{n}+A_{n-1} x^{n-1}+\ldots+A_{1} x+A_{0}
$$

where $x$ is a real variable or complex variable, $A_{j}$, for $0<j<n$ and $A_{n} \neq \mathbf{0}$, where 0 is the null matrix or zero matrix in $\mathbb{C}^{N \times N}$.

We recall that the reciprocal gamma function denoted by $\Gamma^{-1}(z)=\frac{1}{\Gamma(z)}$ is an entire function of the complex variable $z$ and thus for any matrix $A$ in $\mathbb{C}^{N \times N}, \Gamma^{-1}(A)$ is a well defined matrix. Furthermore, if $A$ is a matrix such that

$$
A+n I \text { is an invertible matrix for all integers } n \geq 0
$$

where $I$ is the identity matrix in $\mathbb{C}^{N \times N}$, then from $[8,11]$, it follows that

$$
(A)_{n}=A(A+I) \ldots(A+(n-1) I)=\Gamma(A+n I) \Gamma^{-1}(A) ; \quad n \geq 1 ; \quad(A)_{0}=I .
$$

If $k$ is large enough so then for $k>\|B\|$, then we will mention to the following relation which existed in Jódar and Cortés $[14,16]$ in the form

$$
\left\|(B+k I)^{-1}\right\| \leq \frac{1}{k-\|B\|} ; \quad k>\|B\| .
$$

If $A(k, n)$ and $B(k, n)$ are matrices in $\mathbb{C}^{N \times N}$ for $n \geq 0, k \geq 0$, then in an analogous way to the proof of Lemma 11 [8], it follows that

$$
\begin{aligned}
& \sum_{n=0}^{\infty} \sum_{k=0}^{\infty} A(k, n)=\sum_{n=0}^{\infty} \sum_{k=0}^{\left[\frac{1}{2} n\right]} A(k, n-2 k), \\
& \sum_{n=0}^{\infty} \sum_{k=0}^{\infty} B(k, n)=\sum_{n=0}^{\infty} \sum_{k=0}^{n} B(k, n-k) .
\end{aligned}
$$


Similarly to (1.7), we can write

$$
\begin{aligned}
& \sum_{n=0}^{\infty} \sum_{k=0}^{\left[\frac{1}{2} n\right]} A(k, n)=\sum_{n=0}^{\infty} \sum_{k=0}^{\infty} A(k, n+2 k), \\
& \sum_{n=0}^{\infty} \sum_{k=0}^{n} B(k, n)=\sum_{n=0}^{\infty} \sum_{k=0}^{\infty} B(k, n+k) .
\end{aligned}
$$

The hypergeometric matrix function ${ }_{2} F_{1}(A, B ; C ; z)$ has been given in the form

$$
{ }_{2} F_{1}(A, B ; C ; z)=\sum_{k=0}^{\infty} \frac{(A)_{k}(B)_{k}\left[(C)_{k}\right]^{-1}}{n !} z^{k},
$$

for matrices $A, B$ and $C$ in $\mathbb{C}^{N \times N}$ such that $C+n I$ is an invertible matrix for all integers $n \geq 0$ and for $|z|<1$. It has been seen by Jódar and Cortés [14] that the series is absolutely convergent for $|z|=1$ when

$$
m(C)>M(A)+M(B),
$$

where $m(P)$ and $M(P)$ in (1.1) for any matrix $P$ in $\mathbb{C}^{N \times N}$.

The outline of this paper is as follows: In Section 2, we define generalized hypergeometric matrix function and give the convergence properties, matrix differential equation, matrix recurrence relations and integrals. Section 3, we give the hypergeometric matrix function representation, matrix differential equation, matrix recurrence relations, finite summation formulas, an explicit expression, and a different approach to proof of generating functions of the Konhauser matrix polynomials. Finally in Section 4, we give the definition of the Mittag-Leffler and Bessel-Maitland matrix functions and some properties have also been obtained.

\section{GENERALIZED HYPERGEOMETRIC MATRIX FUNCTION}

In this section, the generalized hypergeometric matrix function is defined as in the following.

Definition 1. Let us suppose that $A_{i} ; 1 \leq i \leq p$ and $B_{j} ; 1 \leq j \leq q$ are matrices in $\mathbb{C}^{N \times N}$ such that

$$
B_{j}+n I \text { are invertible matrices for all integers } n \geq 0
$$


where $p$ and $q$ are finite positive integers. Then we define a generalized hypergeometric matrix function as

$$
\begin{aligned}
& { }_{p} F_{q}\left(A_{1}, A_{2}, \ldots, A_{p} ; B_{1}, B_{2}, \ldots, B_{q} ; z\right) \\
& =\sum_{k=0}^{\infty} \frac{z^{k}}{k !}\left(A_{1}\right)_{k}\left(A_{2}\right)_{k} \ldots\left(A_{p}\right)_{k}\left[\left(B_{1}\right)_{k}\right]^{-1}\left[\left(B_{2}\right)_{k}\right]^{-1} \ldots\left[\left(B_{q}\right)_{k}\right]^{-1} \\
& =\sum_{k=0}^{\infty} \frac{z^{k}}{k !} \prod_{i=1}^{p}\left(A_{i}\right)_{k}\left[\prod_{j=1}^{q}\left(B_{j}\right)_{k}\right]^{-1} .
\end{aligned}
$$

For simplicity, we can write

${ }_{p} F_{q}\left(A_{1}, A_{2}, \ldots, A_{p} ; B_{1}, B_{2}, \ldots, B_{q} ; z\right)$ in the form ${ }_{p} F_{q}$,

${ }_{p} F_{q}\left(A_{1} \pm, A_{2}, \ldots, A_{p} ; B_{1}, B_{2}, \ldots, B_{q} ; z\right)$ in the form ${ }_{p} F_{q}\left(A_{1} \pm\right)$,

${ }_{p} F_{q}\left(A_{1}, A_{2} \pm, \ldots, A_{p} ; B_{1}, B_{2}, \ldots, B_{q} ; z\right)$ in the form ${ }_{p} F_{q}\left(A_{2} \pm\right)$,

${ }_{p} F_{q}\left(A_{1}, A_{2}, \ldots, A_{p} \pm ; B_{1}, B_{2}, \ldots, B_{q} ; z\right)$ in the form ${ }_{p} F_{q}\left(A_{p} \pm\right)$,

${ }_{p} F_{q}\left(A_{1}, A_{2}, \ldots, A_{p} ; B_{1} \pm, B_{2}, \ldots, B_{q} ; z\right)$ in the form ${ }_{p} F_{q}\left(B_{1} \pm\right)$,

${ }_{p} F_{q}\left(A_{1}, A_{2}, \ldots, A_{p} ; B_{1}, B_{2}, \ldots, B_{q} \pm ; z\right)$ in the form ${ }_{p} F_{q}\left(B_{q} \pm\right)$,

$\frac{z^{k}}{k !}\left(A_{1}\right)_{k}\left(A_{2}\right)_{k} \ldots\left(A_{p}\right)_{k}\left[\left(B_{1}\right)_{k}\right]^{-1}\left[\left(B_{2}\right)_{k}\right]^{-1} \ldots\left[\left(B_{q}\right)_{k}\right]^{-1}$ in the form $U_{k}(z)$, and $\frac{1}{k !}\left(A_{1}\right)_{k}\left(A_{2}\right)_{k} \ldots\left(A_{p}\right)_{k}\left[\left(B_{1}\right)_{k}\right]^{-1}\left[\left(B_{2}\right)_{k}\right]^{-1} \ldots\left[\left(B_{q}\right)_{k}\right]^{-1}$ in the form $U_{k}$.

Now, we are going to study the convergence properties of the generalized hypergeometric matrix function. If $k$ is large enough so that $k>\left\|B_{j}\right\|$, then by the perturbation lemma, see [14], it follows

$$
\left\|\left(\frac{1}{k} B_{j}+I\right)^{-1}\right\| \leq \frac{1}{1-\frac{1}{k}\left\|B_{j}\right\|}=\frac{k}{k-\left\|B_{j}\right\|} ; 1 \leq j \leq q
$$

and

$$
\begin{aligned}
\left\|\left(B_{j}+k I\right)^{-1}\right\| & =\left\|\frac{1}{k}\left(\frac{1}{k} B_{j}+I\right)^{-1}\right\|=\frac{1}{k}\left\|\left(\frac{1}{k} B_{j}+I\right)^{-1}\right\| \\
& \leq \frac{1}{k-\left\|B_{j}\right\|} ; \quad k>\left\|B_{j}\right\| ; 1 \leq j \leq q .
\end{aligned}
$$

Let's consider the expression

$$
T_{j}(k)=\left\|B_{j}^{-1}\right\|\left\|\left(B_{j}+I\right)^{-1}\right\| \ldots\left\|\left(B_{j}+(k-1) I\right)^{-1}\right\|
$$

for $k \geq 1$ and $1 \leq j \leq q$.

From (1.5), we obtain

$$
\left\|\left(A_{i}\right)_{k}\right\| \leq\left(\left\|A_{i}\right\|\right)_{k} ; 1 \leq i \leq p .
$$


For $k>\left\|B_{j}\right\|$, by using (2.3), (2.4) and (2.5) can be written

$$
\begin{aligned}
& \left\|\frac{z^{k}}{k !}\left(A_{1}\right)_{k}\left(A_{2}\right)_{k} \ldots\left(A_{p}\right)_{k}\left[\left(B_{1}\right)_{k}\right]^{-1}\left[\left(B_{2}\right)_{k}\right]^{-1} \ldots\left[\left(B_{q}\right)_{k}\right]^{-1}\right\| \\
& \leq \frac{1}{k !}\left|z^{k}\right|\left\|\left(A_{1}\right)_{k}\right\|\left\|\left(A_{2}\right)_{k}\right\| \ldots\left\|\left(A_{p}\right)_{k}\right\| T_{1}(k) T_{2}(k) \ldots T_{q}(k) \\
& \leq \frac{1}{k !}\left|z^{k}\right|\left(\left\|A_{1}\right\|\right)_{k}\left(\left\|A_{2}\right\|\right)_{k} \ldots\left(\left\|A_{p}\right\|\right)_{k} T_{1}(k) T_{2}(k) \ldots T_{q}(k) .
\end{aligned}
$$

Now, we will investigate the convergence of the following series

$$
\sum_{k=0}^{\infty} \frac{1}{k !}\left|z^{k}\right|\left(\left\|A_{1}\right\|\right)_{k}\left(\left\|A_{2}\right\|\right)_{k} \ldots\left(\left\|A_{p}\right\|\right)_{k} T_{1}(k) T_{2}(k) \ldots T_{q}(k) .
$$

By the ratio test and using the relation (1.6), one gets

$$
\begin{aligned}
& \lim _{k \rightarrow \infty}\left|\frac{\left(\left\|A_{1}\right\|\right)_{k+1}\left(\left\|A_{2}\right\|\right)_{k+1} \ldots\left(\left\|A_{p}\right\|\right)_{k+1} T_{1}(k+1) T_{2}(k+1) \ldots T_{q}(k+1) k !}{\left(\left\|A_{1}\right\|\right)_{k}\left(\left\|A_{2}\right\|\right)_{k} \ldots\left(\left\|A_{p}\right\|\right)_{k} T_{1}(k) T_{2}(k) \ldots T_{q}(k)(k+1) !} \frac{z^{k+1}}{z^{k}}\right| \\
& \leq \lim _{k \rightarrow \infty}\left|\frac{\left(\left\|A_{1}\right\|+k\right) \ldots\left(\left\|A_{p}\right\|+k\right)\left\|\left(B_{1}+k I\right)^{-1}\right\| \ldots\left\|\left(B_{q}+k I\right)^{-1}\right\|}{z^{k+1}} \frac{\left.z^{k}+1\right)}{z^{k}}\right| \\
& \leq \lim _{k \rightarrow \infty} \frac{\left(\left\|A_{1}\right\|+k\right)\left(\left\|A_{2}\right\|+k\right) \ldots\left(\left\|A_{p}\right\|+k\right)}{\left(k-\left\|B_{1}\right\|\right)\left(k-\left\|B_{2}\right\|\right) \ldots\left(k-\left\|B_{q}\right\|\right)(k+1)}|z| .
\end{aligned}
$$

The last limit shows that

(1) If $p \leq q$, then the power series (2.2) converges for all finite $z$.

(2) If $p=q+1$, then the power series (2.2) is convergent for $|z|<1$ and diverges for $|z|>1$.

(3) If $p>q+1$, then the power series (2.2) diverges for all $z, z \neq 0$.

In analogous to Theorem 3 in [14], we can state the following:

Theorem 1. (1) If $p=q+1$, then the power series (2.2) is absolutely convergent for $|z|=1$ when

$$
\sum_{j=1}^{q} m\left(B_{j}\right)>\sum_{j=1}^{p} M\left(A_{j}\right) .
$$

(2) If $p=q+1$, then the power series (2.2) is conditionally convergent for $|z|=$ $1, z \neq 1$ when

$$
\sum_{j=1}^{p} M\left(A_{j}\right)-1<\sum_{j=1}^{q} m\left(B_{j}\right) \leq \sum_{j=1}^{p} M\left(A_{j}\right) .
$$

(3) If $p=q+1$, then the power series (2.2) is diverges for $|z|=1$ when

$$
\sum_{j=1}^{q} m\left(B_{j}\right) \leq \sum_{j=1}^{p} M\left(A_{j}\right)-1
$$


where $M\left(A_{j}\right)$ and $m\left(B_{j}\right)$ are defined in (1.1).

Consider the differential operator $\theta=z \frac{d}{d z}, D_{z}=\frac{d}{d z}, \theta z^{k}=k z^{k}$. For these matrices which commute with one another, thus we have

$$
\begin{aligned}
\theta \prod_{j=1}^{q}\left(\theta I+B_{j}-I\right)_{p} F_{q} & =\sum_{k=1}^{\infty} \frac{k z^{k}}{k !} \prod_{j=1}^{q}\left(k I+B_{j}-I\right) \prod_{i=1}^{p}\left(A_{i}\right)_{k}\left[\prod_{j=1}^{q}\left(B_{j}\right)_{k}\right]^{-1} \\
& =\sum_{k=1}^{\infty} \frac{z^{k}}{(k-1) !} \prod_{i=1}^{p}\left(A_{i}\right)_{k}\left[\prod_{j=1}^{q}\left(B_{j}\right)_{k-1}\right]^{-1}
\end{aligned}
$$

Replace $k$ by $k+1$, we have

$$
\begin{aligned}
\theta \prod_{j=1}^{q}\left(\theta I+B_{j}-I\right)_{p} F_{q} & =\sum_{k=0}^{\infty} \frac{z^{k+1}}{k !} \prod_{i=1}^{p}\left(A_{i}\right)_{k+1}\left[\prod_{j=1}^{q}\left(B_{j}\right)_{k}\right]^{-1} \\
& =z \prod_{i=1}^{p}\left(\theta I+A_{i}\right)_{p} F_{q} .
\end{aligned}
$$

Thus, we have shown that ${ }_{p} F_{q}$ is a solution of the following matrix differential equation

$$
\left[\theta \prod_{j=1}^{q}\left(\theta I+B_{j}-I\right)-z \prod_{i=1}^{p}\left(\theta I+A_{i}\right)\right]{ }_{p} F_{q}=\mathbf{0}
$$

where $\mathbf{0}$ is the null matrix in $\mathbb{C}^{N \times N}$. These results are summarized below.

Theorem 2. Let $A_{i} ; 1 \leq i \leq p$ and $B_{j} ; 1 \leq j \leq q$ be matrices in $\mathbb{C}^{N \times N}$ satisfying (2.1) and all matrices are commutative. The generalized hypergeometric matrix function satisfies the matrix differential equation in (2.10).

The solution is valid for all finite $z$ when $p \leq q$. If $p=q+1$, the solution is valid in $|z|<1$. We are not concerning ourselves with the case $p>q+1$, when the series for $p F_{q}$ has a zero radius of convergence unless the series terminates.

If $p<q+1$, the matrix differential equation (2.10) of the preceding section is a linear matrix differential equation of order $(q+1)$. We have, in the neighborhood of the origin, one solution,

$$
\begin{aligned}
w_{0} & ={ }_{p} F_{q}={ }_{p} F_{q}\left(A_{1}, A_{2}, \ldots, A_{p} ; B_{1}, B_{2}, \ldots, B_{q} ; z\right) \\
& =\sum_{k=0}^{\infty} \frac{z^{k}}{n !}\left(A_{1}\right)_{k}\left(A_{2}\right)_{k} \ldots\left(A_{p}\right)_{k}\left[\left(B_{1}\right)_{k}\right]^{-1}\left[\left(B_{2}\right)_{k}\right]^{-1} \ldots\left[\left(B_{q}\right)_{k}\right]^{-1}
\end{aligned}
$$

Naturally we wish to obtain $q$ other solutions near $z=0$.

Let $D_{0}$ be the complex plane cut along the negative real axis, and let us denote $z^{I-B_{m}}=\exp \left(\left(I-B_{m}\right) \log z\right)$ where log represents the principal logarithm [25]. We 
shall now verify that $w_{m}$ satisfies equation (2.10) for all commutative matrices. Let us seek a solution, as before, for $m$ any one of the integers $1,2,3, \ldots, q$, of the form

$$
\begin{aligned}
w_{m}= & z^{I-B_{m}}{ }_{p} F_{q}\left(A_{1}-B_{m}+I, A_{2}-B_{m}+I, \ldots, A_{p}-B_{m}+I, B_{1}-B_{m}+I, \ldots,\right. \\
& \left.B_{m-1}-B_{m}+I, 2 I-B_{m}, B_{m+1}-B_{m}+I, \ldots, B_{q}-B_{m}+I ; z\right) \\
= & \sum_{k=0}^{\infty} z^{(k+1) I-B_{m}}\left[\prod_{i=1}^{p}\left(A_{i}-B_{m}+I\right)_{k}\right]\left[\prod_{j=1}^{q}\left(B_{j}-B_{m}+I\right)_{k}\right]^{-1}\left[\left(2 I-B_{m}\right)_{k}\right]^{-1} \\
& |z|<1, z \in D_{0} .
\end{aligned}
$$

Whenever, in addition to the above restrictions, $B_{j}$ hasn't any positive integer eigenvalue $(j=1,2, \ldots, q)$, then the linear combination (2.11) is the general solution of equation (2.10) around $z=0$. Note also that if $p<q$, then the series for $w_{m}$; $m=0,1,2, \ldots, q$, converges for all finite $z$ and that for $p=q+1$, the series for $w_{m}$ converges for $|z|<1$. From (2.11), we get

$$
\begin{aligned}
\theta & \prod_{j=1}^{q}\left(\theta I+B_{j}-I\right) w_{m} \\
= & \sum_{k=0}^{\infty} z^{(k+1) I-B_{m}}\left((k+1) I-B_{m}\right)\left[\prod_{j=1}^{q}\left((k+1) I-B_{m}+B_{j}-I\right)\right] \\
& {\left[\prod_{i=1}^{p}\left(A_{i}-B_{m}+I\right)_{k}\right]\left[\prod_{j=1}^{q}\left(B_{j}-B_{m}+I\right)_{k}\right]^{-1}\left[\left(2 I-B_{m}\right)_{k}\right]^{-1} . }
\end{aligned}
$$

Now $\left(B_{j}-B_{m}+k I\right)$ is the last factor in the product $\left(B_{j}-B_{m}+I\right)_{k}$ and $(k I+I-$ $\left.B_{m}\right)$ is the last factor in the product $\left(2 I-B_{m}\right)_{k}$.

Hence,

$$
\begin{aligned}
\theta \prod_{j=1}^{q}\left(\theta I+B_{j}-I\right) w_{m}= & \sum_{k=1}^{\infty} z^{(k+1) I-B_{m}}\left[\prod_{i=1}^{p}\left(A_{i}-B_{m}+I\right)_{k}\right] \\
& {\left[\prod_{j=1}^{q}\left(B_{j}-B_{m}+I\right)_{k-1}\right]^{-1}\left[\left(2 I-B_{m}\right)_{k-1}\right]^{-1} }
\end{aligned}
$$


Next, with a shift of index from $k$ to $(k+1)$, we get

$$
\begin{aligned}
\theta & \prod_{j=1}^{q}\left(\theta I+B_{j}-I\right) w_{m} \\
= & \sum_{k=0}^{\infty} z^{(k+2) I-B_{m}}\left[\prod_{i=1}^{p}\left(A_{i}-B_{m}+I\right)_{k+1}\right]\left[\prod_{j=1}^{q}\left(B_{j}-B_{m}+I\right)_{k}\right]^{-1}\left[\left(2 I-B_{m}\right)_{k}\right]^{-1} \\
= & z \sum_{k=0}^{\infty} z^{(k+1) I-B_{m}}\left[\prod_{i=1}^{p}\left(A_{i}-B_{m}+(k+1) I\right)\right]\left[\prod_{i=1}^{p}\left(A_{i}-B_{m}+I\right)_{k}\right] \\
& {\left[\prod_{j=1}^{q}\left(B_{j}-B_{m}+I\right)_{k}\right]^{-1}\left[\left(2 I-B_{m}\right)_{k}\right]^{-1} }
\end{aligned}
$$

Therefore, we have

$$
\theta \prod_{j=1}^{q}\left(\theta I+B_{j}-I\right) w_{m}=z \prod_{i=1}^{p}\left(\theta I+A_{i}\right) w_{m}
$$

so that, $w_{m} ; m=1,2, \ldots, q$, satisfies the matrix differential equation of (2.11) for $z \in D_{0}$ and $|z|<1$.

Assume $A_{i}(i=1,2, \ldots, p)$ and $B_{j}(j=1,2, \ldots, q)$ haven't any integer eigenvalues are these matrices which commute with one another. The relation $A_{i}\left(A_{i}+I\right)_{k}=$ $\left(A_{i}+k I\right)\left(A_{i}\right)_{k}$ together with the definitions of the matrix contiguous relations, yield the formulas:

$$
\begin{aligned}
& { }_{p} F_{q}\left(A_{1}+\right)=\sum_{k=0}^{\infty} \frac{z^{k}}{n !}\left(A_{1}+I\right)_{k}\left(A_{2}\right)_{k} \ldots\left(A_{p}\right)_{k}\left[\left(B_{1}\right)_{k}\right]^{-1}\left[\left(B_{2}\right)_{k}\right]^{-1} \ldots\left[\left(B_{q}\right)_{k}\right]^{-1} \\
& =\sum_{k=0}^{\infty} \frac{z^{k}}{n !}\left(A_{1}+k I\right)\left(A_{1}\right)^{-1}\left(A_{1}\right)_{k}\left(A_{2}\right)_{k} \ldots\left(A_{p}\right)_{k}\left[\left(B_{1}\right)_{k}\right]^{-1}\left[\left(B_{2}\right)_{k}\right]^{-1} \ldots\left[\left(B_{q}\right)_{k}\right]^{-1} \\
& =\sum_{k=0}^{\infty}\left(A_{1}+k I\right)\left(A_{1}\right)^{-1} U_{k}(z) .
\end{aligned}
$$


Similarly, we get

$$
\begin{aligned}
p F_{q}\left(A_{i}+\right) & =\left(A_{i}\right)^{-1} \sum_{k=0}^{\infty}\left(A_{i}+k I\right) U_{k}(z), \\
p F_{q}\left(A_{i}-\right) & =\left(A_{i}-I\right) \sum_{k=0}^{\infty}\left(A_{i}+(k-1) I\right)^{-1} U_{k}(z), \\
p F_{q}\left(B_{j}+\right) & =\left(B_{j}\right) \sum_{k=0}^{\infty}\left(B_{j}+k I\right)^{-1} U_{k}(z) \\
F_{q}\left(B_{j}-\right) & =\left(B_{j}-I\right)^{-1} \sum_{k=0}^{\infty}\left(B_{j}+(k-1) I\right) U_{k}(z) .
\end{aligned}
$$

For all integers $m \geq 1$, we deduce that

$$
\begin{aligned}
& { }_{p} F_{q}\left(A_{i}+m I\right)=\prod_{r=1}^{m}\left(A_{i}+(r-1) I\right)^{-1} \sum_{k=0}^{\infty} \prod_{r=1}^{m}\left(A_{i}+(k+r-1) I\right) U_{k}(z), \\
& { }_{p} F_{q}\left(A_{i}-m I\right)=\prod_{r=1}^{m}\left(A_{i}-r I\right) \sum_{k=0}^{\infty} \prod_{r=1}^{m}\left(A_{i}+(k-r) I\right)^{-1} U_{k}(z), \\
& { }_{p} F_{q}\left(B_{j}+m I\right)=\prod_{r=1}^{m}\left(B_{j}+(r-1) I\right) \sum_{k=0}^{\infty} \prod_{r=1}^{m}\left(B_{j}+(k+r-1) I\right)^{-1} U_{k}(z)
\end{aligned}
$$

and

$$
{ }_{p} F_{q}\left(B_{j}-m I\right)=\prod_{r=1}^{m}\left(B_{j}-r I\right)^{-1} \sum_{k=0}^{\infty} \prod_{r=1}^{m}\left(B_{j}+(k-r) I\right) U_{k}(z)
$$

Using the differential operator $\theta=z \frac{d}{d z}$, we see that

$$
\left(\theta I+A_{i}\right)_{p} F_{q}=\sum_{k=0}^{\infty}\left(A_{i}+k I\right) U_{k}(z) .
$$

Hence, with the aid of (2.14),

$$
\left(\theta I+A_{i}\right)_{p} F_{q}=A_{i p} F_{q}\left(A_{i}+\right) ; i=1,2, \ldots, p .
$$

Similarly, we have

$$
\left(\theta I+B_{j}-I\right)_{p} F_{q}=\left(B_{j}-I\right)_{p} F_{q}\left(B_{j}-\right) ; j=1,2, \ldots, q .
$$

The result is the set of simple relations,

$$
\left(A_{1}-A_{i}\right)_{p} F_{q}=A_{1 p} F_{q}\left(A_{1}+\right)-A_{i p} F_{q}\left(A_{i}+\right) ; i=2,3, \ldots, p
$$


and

$$
\left(A_{1}-B_{j}+I\right)_{p} F_{q}=A_{1 p} F_{q}\left(A_{1}+\right)-\left(B_{j}-I\right)_{p} F_{q}\left(B_{j}-\right) ; j=1,2, \ldots, q .
$$

It is well known that

$$
\begin{gathered}
D_{z p} F_{q}=A_{1} \ldots A_{p}\left(B_{1}\right)^{-1} \ldots\left(B_{q}\right)^{-1}{ }_{p} F_{q}\left(A_{1}+I, \ldots,\right. \\
\left.A_{p}+I ; B_{1}+I, \ldots, B_{q}+I ; z\right) ; D_{z}=\frac{d}{d z}
\end{gathered}
$$

and in general

$$
\begin{aligned}
D_{z p}^{k} F_{q}= & \left(A_{1}\right)_{k}\left(A_{2}\right)_{k} \ldots\left(A_{p}\right)_{k}\left[\left(B_{1}\right)_{k}\right]^{-1}\left[\left(B_{2}\right)_{k}\right]^{-1} \ldots\left[\left(B_{q}\right)_{k}\right]^{-1} \\
& p F_{q}\left(A_{1}+k I, \ldots, A_{p}+k I ; B_{1}+k I, \ldots, B_{q}+k I ; z\right) .
\end{aligned}
$$

\subsection{Integrals involving generalized Hypergeometric matrix function}

The integral representation Eq. (45) in [14] of the generalized hypergeometric matrix function can be extended to obtain the following result:

Theorem 3. Let $A_{i} ; 1 \leq i \leq p$ and $B_{j} ; 1 \leq j \leq q$ be matrices in $\mathbb{C}^{N \times N}$ such that $B_{j}+k I$ are invertible matrices for all integers $k \geq 0$. Suppose that the matrices $A_{1}, B_{1}$ and $B_{1}-A_{1}$ are positive stable matrices. If $p \leq q+1$, then for $|z|<1$ and $A_{i} B_{j}=B_{j} A_{i}$, it follows that

$$
\begin{aligned}
& { }_{p} F_{q}\left(A_{1}, A_{2}, \ldots, A_{p} ; B_{1}, B_{2}, \ldots, B_{q} ; z\right)=\Gamma^{-1}\left(A_{1}\right) \Gamma^{-1}\left(B_{1}-A_{1}\right) \Gamma\left(B_{1}\right) \\
& \int_{0}^{1} t^{A_{1}-I}(1-t)^{B_{1}-A_{1}-I}{ }_{p-1} F_{q-1}\left(A_{2}, \ldots, A_{p} ; B_{2}, \ldots, B_{q} ; z t\right) d t .
\end{aligned}
$$

The proof is similar to the proof of Theorem 5 in [14].

These integral representation can be generalized through the following theorems.

Theorem 4. Let $A_{i} ; 1 \leq i \leq p$ and $B_{j} ; 1 \leq j \leq q$ be matrices in $\mathbb{C}^{N \times N}$ such that $B_{j}+k I$ are invertible matrices for all integers $k \geq 0$. Suppose that $A_{i}, B_{j}$ and $B_{j}-A_{i}$ are positive stable matrices. If $p \leq q+1$, for $|z|<1$

$$
\begin{aligned}
{ }_{p} & F_{q}\left(A_{1}, A_{2}, \ldots, A_{p} ; B_{1}, B_{2}, \ldots, B_{q} ; z\right) \\
= & \Gamma^{-1}\left(A_{i}\right) \Gamma^{-1}\left(B_{j}-A_{i}\right) \Gamma\left(B_{j}\right) \int_{0}^{1} t^{A_{i}-I}(1-t)^{B_{j}-A_{i}-I} \\
& \times{ }_{p-1} F_{q-1}\left(\begin{array}{c}
A_{1}, \ldots, A_{i-1}, A_{i+1} \ldots, A_{p} ; \\
B_{1}, \ldots, B_{j-1}, B_{j+1} \ldots, B_{q}
\end{array} ; t\right) .
\end{aligned}
$$


Proof. By extending Eq. (35) in [14], we have

$$
\left(A_{i}\right)_{n}\left(B_{j}\right)_{n}^{-1}=\Gamma^{-1}\left(A_{i}\right) \Gamma^{-1}\left(B_{j}-A_{i}\right) \Gamma\left(B_{j}\right) \int_{0}^{1} t^{A_{i}+(n-1) I}(1-t)^{B_{j}-A_{i}-I} d t
$$

where $A_{i} B_{j}=B_{j} A_{i}$. Also we have

$$
\begin{aligned}
& { }_{p} F_{q}\left(\begin{array}{c}
A_{1}, A_{2}, \ldots, A_{p} ; \\
B_{1}, B_{2}, \ldots, B_{q} ; z
\end{array}\right) \\
& =\sum_{k=0}^{\infty} \frac{z^{k}}{k !}\left(A_{1}\right)_{k} \ldots\left(A_{i}\right)_{k} \ldots\left(A_{p}\right)_{k}\left[\left(B_{1}\right)_{k}\right]^{-1} \ldots\left[\left(B_{j}\right)_{k}\right]^{-1} \ldots\left[\left(B_{q}\right)_{k}\right]^{-1} \\
& =\sum_{k=0}^{\infty} \frac{z^{k}}{k !}\left(A_{1}\right)_{k} \ldots\left(A_{i-1}\right)_{k}\left(A_{i+1}\right)_{k} \ldots\left(A_{p}\right)_{k}\left[\left(B_{1}\right)_{k}\right]^{-1} \ldots\left[\left(B_{j-1}\right)_{k}\right]^{-1}\left[\left(B_{j+1}\right)_{k}\right]^{-1} \\
& \ldots\left[\left(B_{q}\right)_{k}\right]^{-1} \times \Gamma^{-1}\left(A_{i}\right) \Gamma^{-1}\left(B_{j}-A_{i}\right) \Gamma\left(B_{j}\right) \int_{0}^{1} t^{A_{i}+(n-1) I}(1-t)^{B_{j}-A_{i}-I} d t \\
& =\Gamma^{-1}\left(A_{i}\right) \Gamma^{-1}\left(B_{j}-A_{i}\right) \Gamma\left(B_{j}\right) \int_{0}^{1} t^{A_{i}-I}(1-t)^{B_{j}-A_{i}-I} \\
& \times \sum_{k=0}^{\infty} \frac{(z t)^{k}}{k !}\left(A_{1}\right)_{k} \ldots\left(A_{i-1}\right)_{k}\left(A_{i+1}\right)_{k} \ldots\left(A_{p}\right)_{k} \\
& {\left[\left(B_{1}\right)_{k}\right]^{-1} \ldots\left[\left(B_{j-1}\right)_{k}\right]^{-1}\left[\left(B_{j+1}\right)_{k}\right]^{-1} \ldots\left[\left(B_{q}\right)_{k}\right]^{-1} d t} \\
& =\Gamma^{-1}\left(A_{i}\right) \Gamma^{-1}\left(B_{j}-A_{i}\right) \Gamma\left(B_{j}\right) \int_{0}^{1} t^{A_{i}-I}(1-t)^{B_{j}-A_{i}-I}{ }_{p-1} F_{q-1} d t,
\end{aligned}
$$

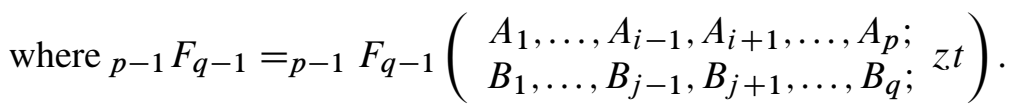

Now, we are going to evaluate some matrix integrals involving generalized Hypergeometric matrix function. Let us consider the integral representation

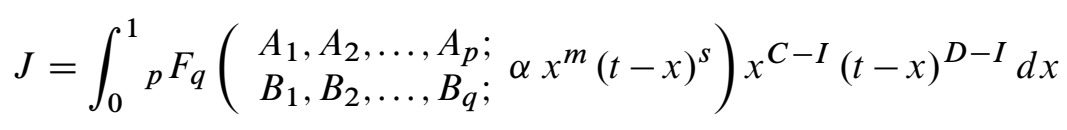

where $C, D$ and $C+D$ are positive stable matrices, $C D=D C$, and $m, s \in \mathbb{N}$ and $B_{j} C=C B_{j}, B_{j} D=D B_{j}$ for $j=1,2, \ldots, m$. 
To evaluate the integral representation, let us substitute $x=t y$, which gives

$$
\begin{aligned}
J= & \int_{0}^{1}{ }_{p} F_{q}\left(A_{1}, A_{2}, \ldots, A_{p} ; B_{1}, B_{2}, \ldots, B_{q} ; \alpha t^{m+s} y^{m}(1-y)^{s}\right) \\
& t^{C+D-I} y^{C-I}(1-y)^{D-I} d y \\
= & \int_{0}^{1} \sum_{k=0}^{\infty} \frac{t^{k(m+s)} \alpha^{k}}{k !} \prod_{i=1}^{p}\left(A_{i}\right)_{k}\left[\prod_{j=1}^{q}\left(B_{j}\right)_{k}\right]^{-1} \\
& y^{C+(m k-1) I}(1-y)^{D+(s k-1) I} t^{C+D-I} d y \\
= & \sum_{k=0}^{\infty} \frac{\alpha^{k} t^{k(m+s)}}{k !} \prod_{i=1}^{p}\left(A_{i}\right)_{k}\left[\prod_{j=1}^{q}\left(B_{j}\right)_{k}\right]^{-1} B(C+m k I, D+s k I) t^{C+D-I} .
\end{aligned}
$$

We can write

$$
\begin{aligned}
& B(C+m k I, D+s k I)=\Gamma(C+m k I) \Gamma(D+s k I) \Gamma^{-1}(C+D+k(m+s) I) \\
& =\Gamma(C) \Gamma(D) \Gamma^{-1}(C+D)(C)_{m k}(D)_{s k}\left[(C+D)_{(m+s) k}\right]^{-1} .
\end{aligned}
$$

Note that we have used here the following elementary result:

$$
(A)_{k m}=k^{k m}\left(\frac{A}{k}\right)_{m}\left(\frac{A+I}{k}\right)_{m} \ldots\left(\frac{A+(k-1) I}{k}\right)_{m} .
$$

By using (2.23), we have

$$
\begin{aligned}
B(C+m k I, D+s k I) & =\frac{m^{m k} s^{s k}}{(m+s)^{(m+s) k}} B(C, D) \prod_{u=1}^{m}\left(\frac{C+(u-1) I}{m}\right)_{k} \\
& \times \prod_{w=1}^{s}\left(\frac{D+(w-1) I}{s}\right)_{k}\left[\prod_{v=1}^{m+s}\left(\frac{C+D+(v-1) I}{m+s}\right)_{k}\right]^{-1} .
\end{aligned}
$$

Hence we get

$$
\begin{aligned}
J & =\sum_{k=0}^{\infty} \frac{m^{m k} s^{s k} c \alpha^{k} t^{k(m+s)}}{k !(m+s)^{(m+s) k}} \\
& \times \prod_{i=1}^{p}\left(A_{i}\right)_{k} \prod_{u=1}^{m}\left(\frac{C+(u-1) I}{m}\right)_{k} \prod_{w=1}^{s}\left(\frac{D+(w-1) I}{s}\right)_{k} \\
& \times\left[\prod_{j=1}^{q}\left(B_{j}\right)_{k}\right]^{-1}\left[\prod_{v=1}^{m+s}\left(\frac{C+D+(v-1) I}{m+s}\right)_{k}\right]^{-1}{ }^{C+D-I} B(C, D) \\
= & p+m+s F_{q+m+s}{ }^{C+D-I} B(C, D),
\end{aligned}
$$


where

$$
\begin{aligned}
& { }_{p+m+s} F_{q+m+s}= \\
& p+m+s F_{q+m+s}\left(\begin{array}{c}
A_{1}, \ldots, A_{p}, \frac{C}{m}, \ldots, \frac{C+(m-1) I}{m}, \frac{D}{s}, \ldots, \frac{D+(s-1) I}{s} ; \\
B_{1}, \ldots, B_{q}, \frac{C+D}{m+s}, \ldots, \frac{m^{m} s^{s} \alpha t^{(m+s)}}{m+s} ;
\end{array}\right) .
\end{aligned}
$$

Theorem 5. Let $A_{i} ; 1 \leq i \leq p$ and $B_{j} ; 1 \leq j \leq q$ be matrices in $\mathbb{C}^{N \times N}$ such that $B_{j}+k I$ are invertible matrices for all integers $k \geq 0$. Suppose that $C, D$ and $C+D$ are positive stable matrices such that $C D=D C, B_{j} C=C B_{j}, B_{j} D=D B_{j}$ and $m, s \in \mathbb{N}$. If $p \leq q+1$, then the following integral representation is hold

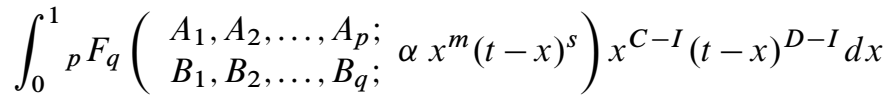

$$
\begin{aligned}
& ={ }_{p+m+s} F_{q+m+s}\left(\begin{array}{c}
A_{1}, \ldots, A_{p}, \frac{C}{m}, \ldots, \frac{C+(m-1) I}{m}, \frac{D}{s}, \ldots, \frac{D+(s-1) I}{s} \\
B_{1}, \ldots, B_{q}, \frac{C+D}{m+s}, \ldots, \frac{C+D+(m+s-1) I}{m+s} ;
\end{array} ; \frac{m^{m} s^{s} \alpha t^{(m+s)}}{(m+s)^{(m+s)}}\right) \\
& { }_{t}^{C+D-I} B(C, D) \text {. }
\end{aligned}
$$

Corollary 1. Let $A_{i} ; 1 \leq i \leq p$ and $B_{j} ; 1 \leq j \leq q$ be matrices in $\mathbb{C}^{N \times N}$ such that $B_{j}+k I$ are invertible matrices for all integers $k \geq 0$. Let $C, D$ and $C+D$ be positive stable matrices such that $C D=D C$ and $m \in \mathbb{N}$. If $p \leq q+1$, then the following integral representation is hold

$$
\begin{aligned}
& \int_{0}^{1}{ }_{p} F_{q}\left(\begin{array}{c}
A_{1}, A_{2}, \ldots, A_{p} ; \\
B_{1}, B_{2}, \ldots, B_{q} ;
\end{array} \alpha x^{m}\right) x^{C-I}(t-x)^{D-I} d x \\
& ={ }_{p+m} F_{q+m}\left(\begin{array}{c}
A_{1}, \ldots, A_{p}, \frac{C}{m}, \ldots, \frac{C+(m-1) I}{m} \\
B_{1}, \ldots, B_{q}, \frac{C+D}{m}, \ldots, \frac{C+D+(m-1) I}{m} ;
\end{array} \alpha t^{m}\right) t^{C+D-I} B(C, D),
\end{aligned}
$$

where $B_{j} C=C B_{j}$ and

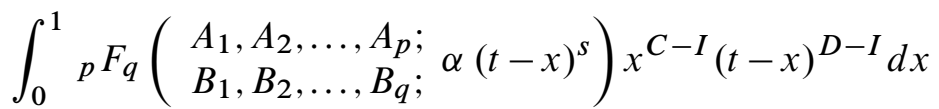

$$
\begin{aligned}
& ={ }_{p+s} F_{q+s}\left(\begin{array}{c}
A_{1}, \ldots, A_{p}, \frac{D}{s}, \ldots, \frac{D+(s-1) I}{s} ; \\
B_{1}, \ldots, B_{q}, \frac{C+D}{s}, \ldots, \frac{C+D+(s-1) I}{s} ;
\end{array} \alpha t^{s}\right) t^{C+D-I} B(C, D),
\end{aligned}
$$

where $B_{j} D=D B_{j}$.

\section{ON KONHAUSER MATRIX POLYNOMIALS}

Let $A$ be a matrix in $\mathbb{C}^{N \times N}$ satisfying the condition

$$
\operatorname{Re}(\mu)>-1, \quad \text { for all eigenvalues } \mu \in \sigma(A)
$$


and $\lambda$ is a complex number with $\operatorname{Re}(\lambda)>0, k \in \mathbb{N}=\{1,2,3, \ldots\}$, and let us consider the Konhauser matrix polynomials $Z_{n}^{(A, \lambda)}(x ; k)$ by means of the relation in the form

$$
Z_{n}^{(A, \lambda)}(x ; k)=\Gamma(k n I+A+I) \sum_{m=0}^{n} \frac{(-1)^{m}(\lambda x)^{m k}}{(n-m) ! m !} \Gamma^{-1}(k m I+A+I)
$$

or

$$
Z_{n}^{(A, \lambda)}(x ; k)=\sum_{m=0}^{n} \frac{(-1)^{m}(\lambda x)^{m k}}{(n-m) ! m !}(A+I)_{k n}\left[(A+I)_{k m}\right]^{-1}
$$

given in [34].

From (3.2) and (2.23), we obtain the hypergeometric matrix function representation

$$
Z_{n}^{(A, \lambda)}(x ; k)=\frac{(A+I)_{k n}}{n !}{ }_{1} F_{k}\left(-n I ; \frac{A+I}{k}, \ldots, \frac{A+k I}{k} ;\left(\frac{\lambda x}{k}\right)^{k}\right)
$$

which can alternatively be used to derive the following properties of the Konhauser matrix polynomials $Z_{n}^{(A, \lambda)}(x ; k)$ by simply specializing those of the generalized hypergeometric matrix function ${ }_{p} F_{q}$ (see [34]).

The Konhauser matrix differential equation (3.4) below follows directly by using (2.10), we set $p=1, q=k, z=\left(\frac{\lambda x}{k}\right)^{k}, \theta=k^{-1} \delta$, where $\delta=x \frac{d}{d x}$, and apply the hypergeometric matrix function representation (3.3), and so we arrive at the following theorem:

Theorem 6. Suppose that $A$ be a matrix in $\mathbb{C}^{N \times N}$ satisfying the condition (3.1). Then the Konhauser matrix polynomials is a solution of the matrix differential equation of order $k+1$ in the form:

$$
\left[\delta \prod_{i=1}^{k}(\delta I+A+(i-k) I)-(\lambda x)^{k}(\delta-k n) I\right] Z_{n}^{(A, \lambda)}(x ; k)=\mathbf{0} .
$$

\subsection{Generating matrix functions for Konhauser matrix polynomials}

The primary purpose of this section is to propose new generating matrix functions of a certain matrix functions in terms of the Konhauser matrix polynomials. 
From (2.23) and (3.2), we have

$$
\begin{aligned}
\sum_{n=0}^{\infty} Z_{n}^{(A, \lambda)}(x ; k)[ & \left.(A+I)_{k n}\right]^{-1} t^{n}=\sum_{n=0}^{\infty} \sum_{m=0}^{n} \frac{(-1)^{m}(\lambda x)^{m k}}{m !(n-m) !}\left[(A+I)_{k m}\right]^{-1} t^{n} \\
& =\sum_{n=0}^{\infty} \sum_{m=0}^{\infty} \frac{(-1)^{m}(\lambda x)^{m k}}{m ! n !}\left[(A+I)_{k m}\right]^{-1} t^{n+m} \\
& =\sum_{n=0}^{\infty} \frac{1}{n !} t^{n} \sum_{m=0}^{\infty} \frac{(-1)^{m}(\lambda x)^{m k}}{m !}\left[(A+I)_{k m}\right]^{-1} t^{m} \\
& =\sum_{n=0}^{\infty} \frac{1}{n !} t^{n} \sum_{m=0}^{\infty} \frac{(-1)^{m}(\lambda x)^{m k}}{k^{k m} m !}\left[\prod_{r=1}^{k}\left(\frac{A+r I}{k}\right)_{m}\right]^{-1} t^{m} \\
& =e^{t}{ }_{0} F_{k}\left(-; \frac{A+I}{k}, \frac{A+2 I}{k}, \ldots, \frac{A+k I}{k} ;-\frac{(\lambda x)^{k} t}{k^{k}}\right) .
\end{aligned}
$$

We can write the new generating matrix function which represents an explicit representation for the Konhauser matrix polynomials in the form

$$
\begin{aligned}
& \sum_{n=0}^{\infty} Z_{n}^{(A, \lambda)}(x ; k)\left[(A+I)_{k n}\right]^{-1} t^{n} \\
&=e^{t}{ }_{0} F_{k}\left(-; \frac{A+I}{k}, \frac{A+2 I}{k}, \ldots, \frac{A+k I}{k} ;-\frac{(\lambda x)^{k} t}{k^{k}}\right) .
\end{aligned}
$$

Now, we consider the double series

$$
\begin{aligned}
\sum_{m=0}^{\infty} u^{m} \sum_{n=0}^{\infty}\left(\begin{array}{c}
m+n \\
n
\end{array}\right)\left[(A+I)_{k(m+n)}\right]^{-1} Z_{m+n}^{(A, \lambda)}(x ; k) t^{n} \\
=\sum_{n=0}^{\infty} \sum_{m=0}^{\infty} u^{m}\left(\begin{array}{c}
m+n \\
n
\end{array}\right)\left[(A+I)_{k(m+n)}\right]^{-1} Z_{m+n}^{(A, \lambda)}(x ; k) t^{n} \\
=\sum_{n=0}^{\infty} \sum_{m=0}^{n} u^{m}\left(\begin{array}{c}
n \\
m
\end{array}\right)\left[(A+I)_{k n}\right]^{-1} Z_{n}^{(A, \lambda)}(x ; k) t^{n-m} \\
=\sum_{n=0}^{\infty}\left[(A+I)_{k n}\right]^{-1} Z_{n}^{(A, \lambda)}(x ; k)(u+t)^{n} \\
=e^{u+t}{ }_{0} F_{k}\left(-; \frac{A+I}{k}, \ldots, \frac{A+k I}{k} ;-\left(\frac{\lambda x}{k}\right)^{k}(u+t)\right) .
\end{aligned}
$$


By (3.5), we have

$$
\begin{aligned}
& \sum_{n, v=0}^{\infty} \frac{\left(-(\lambda x)^{k}\right)^{n}}{n ! v !}\left[(A+I)_{k n}\right]^{-1} \sum_{m=0}^{n+v}\left(\begin{array}{c}
n+v \\
m
\end{array}\right) t^{n+v-m} u^{m} \\
& =\sum_{m=0}^{\infty} u^{m} \sum_{n+v \geq m}\left(\begin{array}{c}
n+v \\
m
\end{array}\right) \frac{\left(-(\lambda x)^{k}\right)^{n} t^{n-m}}{n !}\left[(A+I)_{k n}\right]^{-1} \frac{t^{v}}{v !}
\end{aligned}
$$

On equating the coefficients of $u^{m}$, the new generating matrix function which represents an explicit representation for the Konhauser matrix polynomials gets in the form

$$
\begin{aligned}
& \sum_{n=0}^{\infty}\left(\begin{array}{c}
m+n \\
n
\end{array}\right)\left[(A+I)_{k(m+n)}\right]^{-1} Z_{m+n}^{(A, \lambda)}(x ; k) t^{n} \\
& =\sum_{n=m}\left(\begin{array}{c}
n \\
m
\end{array}\right) \frac{\left(-(\lambda x)^{k}\right)^{n} t^{n-m}}{n !}\left[(A+I)_{k n}\right]^{-1}{ }_{1} F_{1}((n+1) I ;(n-m+1) I ; t)
\end{aligned}
$$

which holds true for every non-negative integer $m$.

Now, we prove a class of new generating matrix functions for the polynomials $Z_{n}^{(A, \lambda)}(x ; k)$ in the following theorem:

Theorem 7. Let $a$ and $b$ are complex constants, not both zero. Then we have

$$
\begin{aligned}
& \sum_{n=0}^{\infty} Z_{n}^{(A, \lambda)}\left(\frac{x}{(a+b n)^{\frac{1}{k}}} ; k\right)(a+b n)^{n}\left[(A+I)_{n k}\right]^{-1} t^{n} \\
& =\frac{e^{a v}}{1-b u}{ }_{0} F_{k}\left(-; \frac{A+I}{k}, \frac{A+2 I}{k}, \ldots, \frac{A+k I}{k} ;-u\left(\frac{\lambda x}{k}\right)^{k}\right)
\end{aligned}
$$

where

$$
u=t e^{b v}
$$

Proof. Starting with the left-hand side of (3.9), we have

$$
\begin{aligned}
& \sum_{n=0}^{\infty} Z_{n}^{(A, \lambda)}\left(\frac{x}{\sqrt[k]{a+b n}} ; k\right)(a+b n)^{n}\left[(A+I)_{n k}\right]^{-1} t^{n} \\
& =\sum_{r=0}^{\infty} \frac{\left(-t x^{k}\right)^{r}}{r !}\left[(A+I)_{k r}\right]^{-1} \sum_{n=0}^{\infty} \frac{(a+b r+b n)^{n}}{n !} t^{n} .
\end{aligned}
$$

Now by setting $f(x)=e^{a x}$ and $\phi(x)=e^{b x}$ in the Lagrange expansion formula

$$
\frac{f(v)}{1-t \phi^{\prime}(v)}=\left.\sum_{n=0}^{\infty} \frac{t^{n}}{n !}\left[D^{n} f(x)(\phi(x))^{n}\right]\right|_{x=0}, u=t \phi(x),
$$


given in [7], we obtain the following elegant result

$$
\frac{e^{a v}}{1-b u}=\sum_{n=0}^{\infty} \frac{(a+b n)^{n}}{n !} t^{n}
$$

where $u$ is given by (3.10).

From (3.11), we have

$$
\begin{aligned}
& \sum_{n=0}^{\infty} Z_{n}^{(A, \lambda)}\left(\frac{x}{\sqrt[k]{a+b n}} ; k\right)(a+b n)^{n}\left[(A+I)_{n k}\right]^{-1} t^{n} \\
& =\sum_{r=0}^{\infty} \frac{\left(-t(\lambda x)^{k}\right)^{r}}{r !}\left[(A+I)_{k r}\right]^{-1} \frac{e^{(a+b r) v}}{1-b u} \\
& =\frac{e^{a v}}{1-b u}{ }_{0} F_{k}\left(-; \frac{A+I}{k}, \frac{A+2 I}{k}, \ldots, \frac{A+k I}{k} ;-u\left(\frac{\lambda x}{k}\right)^{k}\right)
\end{aligned}
$$

and the proof is completed.

For instance, we mention the following particular case. For $a=1$ and $b=0$, our theorem yields the generating matrix function (3.5).

Theorem 8. Let $A$ and $B$ be matrices in $\mathbb{C}^{N \times N}$ such that $A$ satisfies the condition (3.1) and $\lambda$ is a complex number with $\operatorname{Re}(\lambda)>0$. Then we have

$$
\begin{aligned}
& \sum_{n=0}^{\infty}(B)_{n k} Z_{n}^{(A, \lambda)}(x ; k)\left[(A+I)_{n k}\right]^{-1} t^{n}=\sum_{r=0}^{\infty} \frac{\left(-t(\lambda x)^{k}\right)^{r}(B)_{r k}}{r !}\left[(A+I)_{k r}\right]^{-1} \\
& { }_{k} F_{0}\left(\frac{B+r k I}{k}, \frac{B+(r k+1) I}{k}, \ldots, \frac{B+(r k+k-1) I}{k} ;-; t k^{k}\right),\left|t k^{k}\right|<1 .
\end{aligned}
$$

Proof. This can be proved by using equations (2.23) and (3.2).

Substituting $B=A+I$ in equation (3.15), we get the following corollary.

Corollary 2. Let $A$ be a matrix in $\mathbb{C}^{N \times N}$ satisfying the condition (3.1), $\left|t k^{k}\right|<1$ and $\lambda$ is a complex number with $\operatorname{Re}(\lambda)>0$. Then the Konhauser matrix polynomials satisfy the following generating matrix function:

$$
\begin{aligned}
& \sum_{n=0}^{\infty} Z_{n}^{(A, \lambda)}(x ; k) t^{n}=\sum_{r=0}^{\infty} \frac{\left(-t(\lambda x)^{k}\right)^{r}}{r !} \\
& { }_{k} F_{0}\left(\frac{A+(r k+1) I}{k}, \frac{A+(r k+2) I}{k}, \ldots, \frac{A+(r k+k) I}{k} ;-; t k^{k}\right) .
\end{aligned}
$$


Theorem 9. Let $A$ and $B$ be matrices in $\mathbb{C}^{N \times N}$ such that $A-k n I$ satisfies the condition (3.1), $\left|(\lambda x)^{k} t\right|<1,\left|(-k)^{k} t\right|<1$ and $\left|\frac{(-k)^{k} t}{1+(\lambda x)^{k} t}\right|<1$, then the Konhauser matrix polynomials have the linear generating matrix functions

$$
\begin{aligned}
& \sum_{n=0}^{\infty}(B)_{n} Z_{n}^{(A-k n I, \lambda)}(x ; k) t^{n}=\left(1+(\lambda x)^{k} t\right)^{-B} \\
& k+1 F_{0}\left(B,-\frac{A}{k}, \frac{I-A}{k}, \ldots, \frac{(k-1) I-A}{k} ;-; \frac{(-k)^{k} t}{1+(\lambda x)^{k} t}\right)
\end{aligned}
$$

and

$$
\begin{aligned}
& \sum_{n=0}^{\infty} Z_{n}^{(A-k n I, \lambda)}(x ; k) t^{n}=\exp \left(-(\lambda x)^{k} t\right) \\
& { }_{k} F_{0}\left(-\frac{A}{k}, \frac{I-A}{k}, \ldots, \frac{(k-1) I-A}{k} ;-;(-k)^{k} t\right) .
\end{aligned}
$$

Proof. From (1.8), (2.23) and (3.2), we have

$$
\begin{aligned}
\sum_{n=0}^{\infty}(B)_{n} Z_{n}^{(A-k n I, \lambda)}(x ; k) t^{n} \\
=\sum_{n=0}^{\infty} \sum_{m=0}^{n} \frac{(-1)^{m}(\lambda x)^{m k}}{m !(n-m) !}(B)_{n} \Gamma(A+I) \Gamma^{-1}(A+I-k(n-m) I) t^{n} \\
=\sum_{n=0}^{\infty} \sum_{m=0}^{\infty} \frac{(-1)^{m}(\lambda x)^{m k}}{m ! n !}(B)_{n+m} \Gamma(A+I) \Gamma^{-1}(A+I-k n I) t^{n+m} \\
=\sum_{n=0}^{\infty} \sum_{m=0}^{\infty} \frac{(-1)^{m}(\lambda x)^{m k}}{m ! n !}(B)_{n}(B+n I)_{m} \Gamma(A+I) \Gamma^{-1}(A+I-k n I) t^{n+m} \\
=\sum_{n=0}^{\infty} \frac{1}{n !}(B)_{n} \Gamma(A+I) \Gamma^{-1}(A+I-k n I) t^{n} \sum_{m=0}^{\infty} \frac{(-1)^{m}(\lambda x)^{m k}}{m !}(B+n I)_{m} t^{m} \\
=\sum_{n=0}^{\infty} \frac{1}{n !}(B)_{n}(-1)^{k n}(-A)_{k n}\left(1+(\lambda x)^{k} t\right)^{-(B+n I)} t^{n} \\
=\left(1+(\lambda x)^{k} t\right)^{-B} \sum_{n=0}^{\infty} \frac{1}{n !}(B)_{n}(-1)^{k n}(-A)_{k n}\left(\frac{t}{1+(\lambda x)^{k} t}\right)^{n} \\
=\left(1+(\lambda x)^{k} t\right)^{-B} \sum_{n=0}^{\infty} \frac{1}{n !}(B)_{n}(-1)^{k n} k^{k n} \prod_{r=1}^{k}\left(\frac{(r-1) I-A}{k}\right)_{n}\left(\frac{t}{1+(\lambda x)^{k} t}\right)^{n}
\end{aligned}
$$




$$
\begin{aligned}
& =\left(1+(\lambda x)^{k} t\right)^{-B} \sum_{n=0}^{\infty} \frac{1}{n !}(B)_{n}(-k)^{k n} \prod_{r=1}^{k}\left(\frac{(r-1) I-A}{k}\right)_{n}\left(\frac{t}{1+(\lambda x)^{k} t}\right)^{n} \\
& =\left(1+(\lambda x)^{k} t\right)^{-B}{ }_{k+1} F_{0}\left(B,-\frac{A}{k}, \frac{I-A}{k}, \ldots, \frac{(k-1) I-A}{k} ;-; \frac{(-k)^{k} t}{1+(\lambda x)^{k} t}\right) .
\end{aligned}
$$

which gives (3.17). Again, using the relations (1.8), (2.23) and (3.2), we get

$$
\begin{aligned}
\sum_{n=0}^{\infty} Z_{n}^{(A-k n I, \lambda)}(x ; k) t^{n} \\
=\sum_{n=0}^{\infty} \sum_{m=0}^{n} \frac{(-1)^{m}(\lambda x)^{m k}}{m !(n-m) !} \Gamma(A+I) \Gamma^{-1}(A+I-k(n-m) I) t^{n} \\
=\sum_{n=0}^{\infty} \sum_{m=0}^{\infty} \frac{(-1)^{m}(\lambda x)^{m k}}{m ! n !} \Gamma(A+I) \Gamma^{-1}(A+I-k n I) t^{n+m} \\
=\sum_{n=0}^{\infty} \frac{1}{n !} \Gamma(A+I) \Gamma^{-1}(A+I-k n I) t^{n} \sum_{m=0}^{\infty} \frac{(-1)^{m}(\lambda x)^{m k}}{m !} t^{m} \\
=\exp \left(-(\lambda x)^{k} t\right) \sum_{n=0}^{\infty} \frac{1}{n !} \Gamma(A+I) \Gamma^{-1}(A+I-k n I) t^{n} \\
=\exp \left(-(\lambda x)^{k} t\right) \sum_{n=0}^{\infty} \frac{(-1)^{k n}}{n !}(-A)_{k n} t^{n} \\
=\exp \left(-(\lambda x)^{k} t\right) \sum_{n=0}^{\infty} \frac{1}{n !}(-1)^{k n} k^{k n} \prod_{r=1}^{k}\left(\frac{(r-1) I-A}{k}\right)_{n} t^{n} \\
=\exp \left(-(\lambda x)^{k} t\right){ }_{k} F_{0}\left(-\frac{A}{k}, \frac{I-A}{k}, \ldots, \frac{(k-1) I-A}{k} ;-;(-k)^{k} t\right)
\end{aligned}
$$

which leads to (3.18). Thus, the proof is completed.

\subsection{Matrix recurrence relations for Konhauser matrix polynomials}

In this section, we give the matrix recurrence relations for Konhauser matrix polynomials $Z_{n}^{(A, \lambda)}(x ; k)$ together with their proofs.

Theorem 10. Let $A$ be a matrix in $\mathbb{C}^{N \times N}$ satisfying the condition (3.1) and $\lambda$ is a complex number with $\operatorname{Re}(\lambda)>0$. The Konhauser matrix polynomials satisfy

$$
\left(x^{1-k} D_{x}\right)^{r} Z_{n}^{(A, \lambda)}(x ; k)=(-k)^{r} \lambda^{k r} Z_{n-r}^{(A+k r I, \lambda)}(x ; k) ; n \geq r \geq 0 .
$$

Proof. From (2.19) and (2.20), whence, by setting $p=1, q=k, z=\left(\frac{\lambda x}{k}\right)^{k}$, $\frac{d}{d z}=\lambda^{-k}\left(\frac{k}{x}\right)^{k-1} \frac{d}{d x}$, and applying (3.3), we obtain (3.19). 
Theorem 11. Let $A$ and $A-I$ be matrices in $\mathbb{C}^{N \times N}$ satisfying the condition (3.1) and $\lambda$ is a complex number with $\operatorname{Re}(\lambda)>0$. The Konhauser matrix polynomials verify the following properties

$$
x D_{x} Z_{n}^{(A, \lambda)}(x ; k)=(k n I+A) Z_{n}^{(A-I, \lambda)}(x ; k)-A Z_{n}^{(A, \lambda)}(x ; k)
$$

and

$$
\begin{aligned}
Z_{n}^{(A, \lambda)}(x ; k)- & Z_{n}^{(A-I, \lambda)}(x ; k) \\
& =k \Gamma(k n I+A) \Gamma^{-1}(k(n-1) I+A+I) Z_{n-1}^{(A, \lambda)}(x ; k) .
\end{aligned}
$$

Proof. Similarly, from the known results (2.15), (2.16) and (2.18), involving the generalized hypergeometric matrix function (3.3), we readily obtain the following mixed recurrence relations (3.20). It is not difficult to verify that the recurrence relation (3.21) results from (3.20) by eliminating their common term $x D_{x} Z_{n}^{(A, \lambda)}(x ; k)$.

It is worth mentioning that the Konhauser matrix polynomials satisfy the three terms recurrence relationship as given in the following theorem:

Theorem 12. Let $A$ and $A-I$ be matrices in $\mathbb{C}^{N \times N}$ satisfying condition (3.1) and $\lambda$ is a complex number with $\operatorname{Re}(\lambda)>0$. The Konhauser matrix polynomials satisfying the following pure matrix recurrence relations:

$$
k(\lambda x)^{k} Z_{n-1}^{(A+k I, \lambda)}(x ; k)=A Z_{n}^{(A, \lambda)}(x ; k)-(k n I+A) Z_{n}^{(A-I, \lambda)}(x ; k) .
$$

Proof. By derivation with respect to $x$ in (3.20), we have desired relation.

\subsection{Finite summation formulas for Konhauser matrix polynomials}

As usual, we prefer to treat the problem by obtaining the finite summation formulas and then using generating matrix functions techniques.

Theorem 13. Let $A$ be a matrix in $\mathbb{C}^{N \times N}$ satisfying condition (3.1) and $\lambda$ is a complex number with $\operatorname{Re}(\lambda)>0$, then the finite summation formula for Konhauser matrix polynomials are:

$$
Z_{n}^{(A, \lambda)}(x ; k)=\sum_{r=0}^{n} \frac{\lambda^{k r}}{r !}\left(y^{k}-x^{k}\right)^{r} Z_{n-r}^{(A+k r I, \lambda)}(y ; k),
$$

where $A+k r I$ is a matrix in $\mathbb{C}^{N \times N}$ satisfying condition (3.1). 
Proof. Using (3.21) and (1.7), we have

$$
\begin{aligned}
\sum_{n=0}^{\infty} Z_{n}^{(A-k n I, \lambda)}(x ; k) t^{n} & =\exp \left(\lambda^{k} t\left(y^{k}-x^{k}\right)\right) \sum_{n=0}^{\infty} Z_{n}^{(A-k n I, \lambda)}(y ; k) t^{n} \\
& =\sum_{n=0}^{\infty} \sum_{r=0}^{\infty} \frac{\lambda^{k r}}{r !}\left(y^{k}-x^{k}\right)^{r} Z_{n}^{(A-k n I, \lambda)}(y ; k) t^{n+r} \\
& =\sum_{n=0}^{\infty} \sum_{r=0}^{n} \frac{\lambda^{k r}}{r !}\left(y^{k}-x^{k}\right)^{r} Z_{n-r}^{(A-k n I+k r I, \lambda)}(y ; k) t^{n}
\end{aligned}
$$

Comparing the coefficients of $t^{n}$, we get

$$
Z_{n}^{(A-k n I, \lambda)}(x ; k)=\sum_{r=0}^{n} \frac{\lambda^{k r}}{r !}\left(y^{k}-x^{k}\right)^{r} Z_{n-r}^{(A-k n I+k r I, \lambda)}(y ; k) .
$$

Replacing $A$ by $A+k n I$, we obtain (3.23).

Theorem 14. Let $A$ be a matrix in $\mathbb{C}^{N \times N}$ satisfying condition (3.1), then the Konhauser matrix polynomials have the following finite summation formulas:

$$
\begin{aligned}
& Z_{n}^{(A, \lambda)}(x ; k) \\
& =\left(\frac{x}{y}\right)^{k n} \sum_{j=0}^{n}(A+I)_{k n}\left[(A+I)_{k(n-j)}\right]^{-1} \frac{1}{j !}\left(\frac{y^{k}-x^{k}}{x^{k}}\right)^{j} Z_{n-j}^{(A, \lambda)}(y ; k)
\end{aligned}
$$

or equivalently,

$$
\begin{aligned}
& Z_{n}^{(A, \lambda)}(x ; k) \\
& \quad=\left(\frac{x}{y}\right)^{k n} \sum_{j=0}^{n}(A+I)_{k n}\left[(A+I)_{k j}\right]^{-1} \frac{1}{(n-j) !}\left(\frac{y^{k}-x^{k}}{x^{k}}\right)^{n-j} Z_{j}^{(A, \lambda)}(y ; k) .
\end{aligned}
$$

Proof. From (3.5), if we set $t=\left(\frac{y}{k}\right)^{k} z$, we get

$$
\begin{aligned}
& \sum_{n=0}^{\infty}\left[(A+I)_{k n}\right]^{-1} Z_{n}^{(A, \lambda)}(x ; k)\left(\frac{y}{k}\right)^{n k} z^{n} \\
& =\exp \left(\left(\frac{y}{k}\right)^{k} z\right){ }_{0} F_{k}\left(-; \frac{A+I}{k}, \ldots, \frac{A+k I}{k} ;-\left(\frac{\lambda x y}{k^{2}}\right)^{k} z\right)
\end{aligned}
$$


which, on interchanging $x$ and $y$, gives us

$$
\begin{aligned}
& \sum_{n=0}^{\infty}\left[(A+I)_{k n}\right]^{-1} Z_{n}^{(A, \lambda)}(y ; k)\left(\frac{x}{k}\right)^{n k} z^{n} \\
& =\exp \left(\left(\frac{x}{k}\right)^{k} z\right){ }_{0} F_{k}\left(-; \frac{A+I}{k}, \ldots, \frac{A+k I}{k} ;-\left(\frac{\lambda x y}{k^{2}}\right)^{k} z\right) .
\end{aligned}
$$

Using (3.26) and (3.27), it follows that

$$
\begin{aligned}
& \sum_{n=0}^{\infty}\left[(A+I)_{k n}\right]^{-1} Z_{n}^{(A, \lambda)}(x ; k)\left(\frac{y}{k}\right)^{n k} z^{n} \\
& =\exp \left(z\left[\left(\frac{y}{k}\right)^{k}-\left(\frac{x}{k}\right)^{k}\right]\right) \sum_{n=0}^{\infty}\left[(A+I)_{k n}\right]^{-1} Z_{n}^{(A, \lambda)}(y ; k)\left(\frac{x}{k}\right)^{n k} z^{n} .
\end{aligned}
$$

Comparing the coefficients of $z^{n}$, we obtain (3.24). Thus the proof is completed.

Corollary 3. The Konhauser matrix polynomials satisfy the multiplication formula follows:

$Z_{n}^{(A, \lambda)}(\mu x ; k)=\sum_{r=0}^{n}(A+I)_{k n}\left[(A+I)_{k(n-r)}\right]^{-1} \frac{1}{r !} \mu^{k(n-r)}\left(1-\mu^{k}\right)^{r} Z_{n-r}^{(A, \lambda)}(x ; k)$.

Proof. The summation formula (3.24) can indeed be derived directly.

\subsection{Expansions of Konhauser matrix polynomials}

Now, we state and prove the following theorem which will be instrumental in the sequel.

Theorem 15. Let $A$ be a matrix in $\mathbb{C}^{N \times N}$ satisfy the condition (3.1). Then we have

$$
(\lambda x)^{k n} I=\sum_{m=0}^{n} \frac{(-1)^{m} n !}{(n-m) !} Z_{m}^{(A, \lambda)}(x ; k)(A+I)_{k n}\left[(A+I)_{k m}\right]^{-1} .
$$

Proof. From (3.5), we get

$$
\begin{aligned}
& { }_{0} F_{k}\left(-; \frac{A+I}{k}, \frac{A+2 I}{k}, \ldots, \frac{A+k I}{k} ;-\frac{(\lambda x)^{k} t}{k^{k}}\right) \\
& =e^{-t} \sum_{m=0}^{\infty} Z_{m}^{(A, \lambda)}(x ; k)\left[(A+I)_{k m}\right]^{-1} t^{m} .
\end{aligned}
$$


Therefore, we can write the following

$$
\begin{array}{r}
\sum_{n=0}^{\infty} \frac{\left(-(\lambda x)^{k}\right)^{n} t^{n}}{n ! k^{k n}}\left[\left(\frac{A+I}{k}\right)_{n}\right]^{-1}\left[\left(\frac{A+2 I}{k}\right)_{n}\right]^{-1} \ldots\left[\left(\frac{A+k I}{k}\right)_{n}\right]^{-1} \\
=\sum_{n=0}^{\infty} \sum_{m=0}^{n} \frac{(-1)^{n-m}}{(n-m) !} Z_{m}^{(A, \lambda)}(x ; k) t^{n}\left[(A+I)_{k m}\right]^{-1}
\end{array}
$$

Comparing the coefficients of $t^{n}$ in (3.30), we have the proof.

Expansions of Hermite and Legendre matrix polynomials in a series of Konhauser matrix polynomials relevant to the present investigation are summarized following theorem.

Theorem 16. Let $A$ be a matrix in $\mathbb{C}^{N \times N}$ satisfy the condition (3.1) and $\lambda$ is a complex number with $\operatorname{Re}(\lambda)>0$. Hermite, Legendre and Konhauser matrix polynomials satisfy respectively the relation:

$$
\begin{aligned}
H_{n}\left((\lambda x)^{k}, A\right)= & n ! \sum_{m=0}^{n} \sum_{s=0}^{\left[\frac{n-m}{2}\right]} \frac{(-1)^{m+s}(\sqrt{2 A})^{n-2 s}}{(n-m-2 s) ! s !} \\
& Z_{m}^{(A, \lambda)}(x ; k)(A+I)_{k(n-2 s)}\left[(A+I)_{k m}\right]^{-1} .
\end{aligned}
$$

where $H_{n}(x, A)$ stands for Hermite matrix polynomials [8, 11$]$.

$$
\begin{aligned}
P_{n}\left((\lambda x)^{k}, A\right)= & n ! \sum_{m=0}^{n} \sum_{s=0}^{\left[\frac{1}{2}(n-m)\right]} \frac{(-1)^{s+m}\left(\frac{1}{2}\right)_{n-s}(\sqrt{2 A})^{n-2 s}}{s !(n-m-2 s) !} \\
& Z_{m}^{(A, \lambda)}(x ; k)(A+I)_{k(n-2 s)}\left[(A+I)_{k m}\right]^{-1} t^{n} .
\end{aligned}
$$

where $P_{n}(x, A)$ is Legendre matrix polynomials [32].

Proof. We consider the series involving Hermite matrix polynomials

$$
\begin{aligned}
& \sum_{n=0}^{\infty} \frac{t^{n}}{n !} H_{n}\left((\lambda x)^{k}, A\right)=\exp \left((\lambda x)^{k} t \sqrt{2 A}-t^{2} I\right)=\sum_{n=0}^{\infty} \sum_{s=0}^{\infty} \frac{(-1)^{s}(\lambda x)^{k n}(\sqrt{2 A})^{n}}{n ! s !} t^{n+2 s} \\
& =\sum_{n=0}^{\infty} \sum_{s=0}^{\infty} \sum_{m=0}^{n} \frac{(-1)^{s}(\sqrt{2 A})^{n}}{n ! s !} \frac{(-1)^{m} n !}{(n-m) !} Z_{m}^{(A, \lambda)}(x ; k)(A+I)_{k n}\left[(A+I)_{k m}\right]^{-1} t^{n+2 s} \\
& =\sum_{n=0}^{\infty} \sum_{s=0}^{\infty} \sum_{m=0}^{\infty} \frac{(-1)^{s+m}(\sqrt{2 A})^{n+m}}{s ! n !} Z_{m}^{(A, \lambda)}(x ; k)(A+I)_{k(n+m)}\left[(A+I)_{k m}\right]^{-1} t^{n+m+2 s} \\
& =\sum_{n=0}^{\infty} \sum_{m=0}^{\infty} \sum_{s=0}^{\left[\frac{n}{2}\right]} \frac{(-1)^{m+s}(\sqrt{2 A})^{n+m-2 s}}{s !(n-2 s) !} Z_{m}^{(A, \lambda)}(x ; k)(A+I)_{k(n+m-2 s)}\left[(A+I)_{k m}\right]^{-1} t^{n+m}
\end{aligned}
$$


Replacing $n$ by $n-m$, we get

$$
\begin{aligned}
& \sum_{n=0}^{\infty} \frac{t^{n}}{n !} H_{n}\left((\lambda x)^{k}, A\right) \\
& =\sum_{n=0}^{\infty} \sum_{m=0}^{n} \sum_{s=0}^{\left[\frac{n-m}{2}\right]} \frac{(-1)^{m+s}(\sqrt{2 A})^{n-2 s}}{(n-m-2 s) ! s !} Z_{m}^{(A, \lambda)}(x ; k)(A+I)_{k(n-2 s)}\left[(A+I)_{k m}\right]^{-1} t^{n} .
\end{aligned}
$$

Comparing the coefficients of $t^{n}$, we obtain (3.31). In a similar fashion, using the result (3.29) in the generating relation for Legendre matrix polynomials, we get

$$
\begin{aligned}
& \sum_{n=0}^{\infty} \frac{t^{n}}{n !} P_{n}\left((\lambda x)^{k}, A\right) \\
= & \sum_{n=0}^{\infty} \sum_{s=0}^{\left[\frac{1}{2} n\right]} \frac{(-1)^{s}\left(\frac{1}{2}\right)_{n-s}\left((\lambda x)^{k} \sqrt{2 A}\right)^{n-2 s}}{s !(n-2 s) !} t^{n} \\
= & \sum_{n=0}^{\infty} \sum_{s=0}^{\infty} \frac{(-1)^{s}\left(\frac{1}{2}\right)_{n+s}\left((\lambda x)^{k} \sqrt{2 A}\right)^{n}}{s ! n !} t^{n+2 s} \\
= & \sum_{n=0}^{\infty} \sum_{s=0}^{\infty} \frac{(-1)^{s}\left(\frac{1}{2}\right)_{n+s}(\lambda x)^{n k}(\sqrt{2 A})^{n}}{s ! n !} t^{n+2 s} \\
= & \sum_{n, s=0}^{\infty} \sum_{m=0}^{n} \frac{(-1)^{s+m}\left(\frac{1}{2}\right)_{n+s}(\sqrt{2 A})^{n}}{s !(n-m) !} Z_{m}^{(A, \lambda)}(x ; k)(A+I)_{k n}\left[(A+I)_{k m}\right]^{-1} t^{n+2 s} \\
= & \sum_{n, s, m=0}^{\infty} \frac{(-1)^{s+m}\left(\frac{1}{2}\right)_{n+m+s}(\sqrt{2 A})^{n+m}}{s ! n !} Z_{m}^{(A, \lambda)}(x ; k)(A+I)_{k(n+m)} \\
= & \sum_{n, m=0}^{\infty} \sum_{s=0}^{\left[\left(\frac{1}{2} n\right]\right.} \frac{(-1)^{s+m}\left(\frac{1}{2}\right)_{n+m-s}(\sqrt{2 A})^{n+m-2 s}}{s !(n-2 s) !} Z_{m}^{(A, \lambda)}(x ; k)(A+I)_{k(n+m-2 s)} \\
& {\left[(A+I)_{k m}\right]^{-1} t^{n+m} }
\end{aligned}
$$


Replacing $n$ by $n-m$, we get

$$
\begin{aligned}
& \sum_{n=0}^{\infty} \frac{t^{n}}{n !} P_{n}\left((\lambda x)^{k}, A\right) \\
& =\sum_{n=0}^{\infty} \sum_{m=0}^{n} \sum_{s=0}^{\left[\frac{1}{2}(n-m)\right]} \frac{(-1)^{s+m}\left(\frac{1}{2}\right)_{n-s}(\sqrt{2 A})^{n-2 s}}{s !(n-m-2 s) !} Z_{m}^{(A, \lambda)}(x ; k)(A+I)_{k(n-2 s)} \\
& {\left[(A+I)_{k m}\right]^{-1} t^{n} .}
\end{aligned}
$$

Comparing the coefficients of $t^{n}$, we obtain (3.32). Thus the proof is completed.

In conclusion, we shall now show that there exist generating matrix functions between Konhauser matrix polynomials and the various type of Mittag-Leffler and Bessel-Maitland matrix functions, more results could be obtained, but the details are omitted for reasons of brevity.

\section{ApPliCATions}

Notice that the so-called Mittag-Leffler matrix function $E_{k I, A+I}^{B}$ and the BesselMaitland matrix functions $\phi(k I, A+I ; z)$, are indeed the familiar hypergeometric matrix functions ${ }_{1} F_{k}$ and ${ }_{0} F_{k}$ respectively, $k$ is a positive integer. More precisely, we define, for $k=1,2,3, \ldots$,

$$
\begin{aligned}
E_{k I, A+I}^{B}(z) & =\sum_{m=0}^{\infty} \frac{(B)_{m} z^{m}}{m !} \Gamma^{-1}(k m I+A+I) \\
& =\Gamma^{-1}(A+I)_{1} F_{k}\left(B ; \frac{A+I}{k}, \ldots, \frac{A+k I}{k} ; \frac{z}{k^{k}}\right)
\end{aligned}
$$

and

$$
\begin{aligned}
\phi(k I, A+I ; z) & =\sum_{m=0}^{\infty} \frac{z^{m}}{m !} \Gamma^{-1}(k m I+A+I) \\
& =\Gamma^{-1}(A+I)_{0} F_{k}\left(-; \frac{A+I}{k}, \ldots, \frac{A+k I}{k} ; \frac{z}{k^{k}}\right)
\end{aligned}
$$

where $A$ satisfies the condition (3.1).

As a consequence of the definitions (4.1) and (4.2) the following results hold:

Theorem 17. Let $A$ and $B$ be matrices in $\mathbb{C}^{N \times N}$ such that $A$ satisfies the condition (3.1) and $|t|<1$. There hold the following relations:

$$
\sum_{n=0}^{\infty}(B)_{n} \Gamma^{-1}(k n I+A+I) Z_{n}^{(A, \lambda)}(x ; k) t^{n}=(1-t)^{-B} E_{k I, A+I}^{B}\left(\frac{-(\lambda x)^{k} t}{1-t}\right)
$$


and

$$
\sum_{n=0}^{\infty} \Gamma^{-1}(k n I+A+I) Z_{n}^{(A, \lambda)}(x ; k) t^{n}=e^{t} \phi\left(k I, A+I ;-(\lambda x)^{k} t\right) .
$$

Proof. Using (1.8), (4.1) and (3.2), we get another representation for the Konhauser matrix polynomials

$$
\begin{aligned}
& \sum_{n=0}^{\infty}(B)_{n} \Gamma^{-1}(k n I+A+I) Z_{n}^{(A, \lambda)}(x ; k) t^{n} \\
&=\sum_{n=0}^{\infty} \sum_{m=0}^{n} \frac{(-1)^{m}(B)_{n} \Gamma^{-1}(k m I+A+I)(\lambda x)^{k m}}{m !(n-m) !} t^{n} \\
&=\sum_{n=0}^{\infty} \sum_{m=0}^{\infty} \frac{(-1)^{m}(B)_{n+m} \Gamma^{-1}(k m I+A+I)(\lambda x)^{k m}}{m ! n !} t^{n+m} \\
&=\sum_{n=0}^{\infty} \sum_{m=0}^{\infty} \frac{(-1)^{m}(B+m I)_{n}(B)_{m} \Gamma^{-1}(k m I+A+I)(\lambda x)^{k m}}{m ! n !} t^{n+m} \\
&=\sum_{m=0}^{\infty} \sum_{n=0}^{\infty} \frac{(B+m I)_{n}}{n !} t^{n} \frac{(-1)^{m}(B)_{m} \Gamma^{-1}(k m I+A+I)(\lambda x)^{k m}}{m !} t^{m} \\
&= \sum_{m=0}^{\infty}(1-t)^{-(B+m I)} \frac{(-1)^{m}(B)_{m} \Gamma^{-1}(k m I+A+I)(\lambda x)^{k m}}{m !} t^{m} \\
&=(1-t)^{-B} \sum_{m=0}^{\infty} \frac{(B)_{m} \Gamma^{-1}(k m I+A+I)}{m !}\left(\frac{-(\lambda x)^{k} t}{1-t}\right)^{m} \\
&=(1-t)^{-B} E_{k I, A+I}^{B}\left(\frac{-(\lambda x)^{k} t}{1-t}\right) .
\end{aligned}
$$

which gives (4.3). From (1.7), (3.2) and (4.2), we have

$$
\begin{aligned}
e^{t} \phi\left(k I, A+I ;-(\lambda x)^{k} t\right) & =\sum_{n=0}^{\infty} \sum_{m=0}^{\infty} \frac{(-1)^{m}(\lambda x)^{m k}}{n ! m !} t^{n+m} \Gamma^{-1}(k m I+A+I) \\
& =\sum_{n=0}^{\infty} \sum_{m=0}^{n} \frac{(-1)^{m}(\lambda x)^{m k}}{(n-m) ! m !} t^{n} \Gamma^{-1}(k m I+A+I) \\
& =\sum_{n=0}^{\infty} \Gamma^{-1}(k n I+A+I) Z_{n}^{(A, \lambda)}(x ; k) t^{n}
\end{aligned}
$$

Thus the proof is completed. 
In the forthcoming work, we will discuss the properties of these new families of Mittag-Leffler and Bessel-Maitland matrix functions and we will analyze possible developments and applications of the theory. Hence, new results and further applications can be obtained.

\section{ACKNOWLEDGEMENT}

(1) The author expresses their sincere appreciation to Dr. Mohamed Saleh Metwally, (Department of Mathematics, Faculty of Science (Suez), Suez Canal University, Egypt) and Dr. Mahmoud Tawfik Mohamed, (Department of Mathematics, Faculty of Science(New Valley), Assiut University, New Valley, EL-Kharga 72111, Egypt) for their kinds interests, encouragements, helps, suggestions, comments them and the investigations for this series of papers.

(2) The author would like to thank the referees for their valuable comments and suggestions which have led to the better presentation of the paper.

\section{REFERENCES}

[1] R. Aktaş, B. Çekim, and R. Şahin, "The matrix version for the multivariable Humbert polynomials," Miskolc Math. Notes, vol. 13, no. 2, pp. 197-208, 2012.

[2] A. Altin and B. Çekim, "Generating matrix functions for Chebyshev matrix polynomials of the second kind," Hacettepe J. Math. Statistics, vol. 41, no. 1, pp. 25-32, 2012.

[3] A. Altin and B. Çekim, "Some properties associated with Hermite matrix polynomials," Util. Math., vol. 88, pp. 171-181, 2012.

[4] B. Çekim, A. Altin, and R. Aktaş, "Some relations satisfied by orthogonal matrix polynomials," Hacettepe J. Math. Statistics, vol. 40, no. 2, pp. 241-253, 2011.

[5] B. Çekim, B. Altin, and B. Aktaş, "Some new results for Jacobi matrix polynomials," Filomat, vol. 27, no. 4, pp. 713-719, 2013.

[6] A. Constantine and R. Mairhead, "Partial differential equations for hypergeometric functions of two argument matrices," J. Multivariate Anal., vol. 2, pp. 332-338, 1972, doi: 10.1016/0047259X(72)90020-6.

[7] M. Das, "A generating function for the generalized Bessel polynomials," Amer. Math. Month., vol. 74, pp. 182-183, 1967, doi: 10.2307/2315616.

[8] E. Defez and L. Jódar, "Some applications of Hermite matrix polynomials series expansions," $J$. Comput. Appl. Math., vol. 99, pp. 105-117, 1998, doi: 10.1016/S0377-0427(98)00149-6.

[9] N. Dunford and J. Schwartz, Linear Operators, part I, General Theory. New York: Interscience Publishers, INC., 1957, vol. I.

[10] A. James, Special Functions of Matrix and Single Argument in Statistics in Theory and Application of Special Functions. New York: R.A. Askey (Ed) Academic Press, 1975.

[11] L. Jódar and R. Company, "Hermite matrix polynomials and second order matrix differential equations," Approx. Theory Appl., vol. 12, no. 2, pp. 20-30, 1996.

[12] L. Jódar, R. Company, and E. Navarro, "Laguerre matrix polynomials and system of secondorder differential equations," Appl. Numer. Math., vol. 15, pp. 53-63, 1994, doi: 10.1016/01689274(94)00012-3.

[13] L. Jódar, R. Company, and E. Ponsoda, "Orthogonal matrix polynomials and systems of second order differential equations,” Differ. Equ. Dyn. Syst., vol. 3, pp. 269-288, 1996.

[14] L. Jódar and J. Cortés, “On the hypergeometric matrix function,” J. Comput. Appl. Math., vol. 99, pp. 205-217, 1998, doi: 10.1016/S0377-0427(98)00158-7. 
[15] L. Jódar and J. Cortés, "Some properties of Gamma and Beta matrix functions," Appl. Math. Lett., vol. 11, no. 1, pp. 89-93, 1998.

[16] L. Jódar and J. Cortés, "Closed form general solution of the hypergeometric matrix differential equation," Mathematical Computer Modelling, vol. 32, pp. 1017-1028, 2000, doi: 10.1016/S0895-7177(00)00187-4.

[17] L. Jódar and E. Defez, "On Hermite matrix polynomials and Hermite matrix functions," Approx. Theory Appl., vol. 14, pp. 36-48, 1998.

[18] L. Jódar and J. Sastre, “On Laguerre matrix polynomials," Util. Math., vol. 53, pp. 37-48, 1998.

[19] L. Kargin and V. Kurt, "Some relations on Hermite matrix polynomials," Mathematical Computational Applications, vol. 18, no. 3, pp. 323-329, 2013.

[20] J. Konhauser, "Some properties of biorthogonal polynomials," J. Mathematical Anal. Applications, vol. 11, pp. 242-260, 1965, doi: 10.1016/0022-247X(65)90085-5.

[21] M. Metwally, M. Mohamed, and A. Shehata, "On Hermite-Hermite matrix polynomials," Math. Bohemica, vol. 133, pp. 421-434, 2008.

[22] R. Muirhead, "Systems of partial differential equations for hypergeometric functions of matrix argument," Annals Mathematical Statistics, vol. 41, pp. 991-1001, 1970, doi: 10.1214/aoms/1177696975.

[23] S. Preiser, An Investigation of Biorthogonal Polynomials Derivable from Ordinary Differential Equations of the Third Order. New York University: Ph.D. Thesis, 1958.

[24] S. Preiser, "An investigation of biorthogonal polynomials derivable from ordinary differential equations of the third order," J. Mathematical Anal. Applications, vol. 4, pp. 38-64, 1962, doi: 10.1016/0022-247X(62)90028-8.

[25] S. Saks and A. Zygmund, Analytic Functions. Amsterdam: Elsevier, 1971.

[26] J. Sastre and L. Jódar, "On Laguerre matrix polynomials series," Util. Math., vol. 71, pp. 109-130, 2006.

[27] A. Shehata, "On Tricomi and Hermite-Tricomi matrix functions of complex variable," Сотmunications Math. Applications, vol. 2, no. 2-3, pp. 97-109, 2011.

[28] A. Shehata, "A new extension of Gegenbauer matrix polynomials and their properties," Bulletin Inter. Math. Virtual Institute, vol. 2, pp. 29-42, 2012.

[29] A. Shehata, "On pseudo Legendre matrix polynomials," Inter. J. Mathematical Sci. Eng. Applications (IJMSEA), vol. 6, no. VI, pp. 251-258, 2012.

[30] A. Shehata, "On Rice's matrix polynomials," Afrika Matematika, vol. 25, no. 3, pp. 757-777, 2014.

[31] F. Taşdelen, B. Çekim, and R. Aktaş, "On a multivariable extension of Jacobi matrix polynomials," Computers Math. Applications, vol. 61, pp. 2412-2423, 2011, doi: 10.1016/j.camwa.2011.02.019.

[32] L. Upadhyaya and A. Shehata, "On Legendre matrix polynomials and its applications," Inter. Tran. Mathematical Sci. Computer (ITMSC), vol. 4, no. 2, pp. 291-310, 2011.

[33] L. Upadhyaya and A. Shehata, "A new extension of generalized Hermite matrix polynomials," $B$. Malays. Math. Sci. So., vol. 38, no. 1, pp. 165-179, 2015.

[34] S. Varma, B. Çekim, and F. Taşdelen, "On Konhauser matrix polynomials," Ars Combinatoria, vol. 100, pp. 193-204, 2011.

[35] S. Varma and F. Taşdelen, "Biorthogonal matrix polynomials related to Jacobi matrix polynomials," Computers Math. Applications, vol. 62, no. 10, pp. 3663-3668, 2011. 
Author's address

\section{Ayman Shehata}

Department of Mathematics, Faculty of Science, Assiut University, Assiut 71516, Egypt

Department of Mathematics, College of Science and Arts, Unaizah, Qassim University, Qassim, Kingdom of Saudi Arabia.

E-mail address: drshehata2006@yahoo.com 